\title{
Dietary flavonoids as intracellular substrates for an erythrocyte trans-plasma membrane oxidoreductase activity
}

\author{
Mara Fiorani* and Augusto Accorsi \\ Istituto di Chimica Biologica 'Giorgio Fornaini', Università degli Studi di Urbino, Via Saffi 2, 61029 Urbino (PU), Italy \\ (Received 17 December 2004 - Revised 14 April 2005 - Accepted 15 April 2005)
}

\begin{abstract}
The plasma membrane oxidoreductase (PMOR) activity, which mainly utilises ascorbate as intracellular electron donor, represents a major mechanism for cell-dependent reduction of extracellular oxidants and might be an important process used by the erythrocytes to keep a reduced plasma environment. We previously reported that in human erythrocytes, myricetin and quercetin act as intracellular substrates of a PMOR showing a novel mechanism whereby these flavonoids could exert beneficial effects under oxidative stress conditions. Here, we evaluated the ability of different flavonoids (quercetin, myricetin, morin, kaempferol, fisetin, catechin, luteolin, apigenin, acacetin, rutin, taxifolin, naringenin, genistein) and of two in vivo $O$-methylated metabolites of quercetin (isorhamnetin and tamarixetin) to be substrates of PMOR, by comparing their antioxidant capacity (i.e. direct interaction with the oxidant ferricyanide or with the free radical 1,1-diphenyl-2-picryl-hydrazil) with their ability to penetrate the erythrocytes and donate electrons to the PMOR. The results obtained indicate that, although most of the flavonoids display significant antioxidant activities, only those (quercetin, myricetin, fisetin) that combine the cathecol structure of the $\mathrm{B}$ ring (responsible for the reducing activity) with the 2,3 double bond and 4-oxo function of the $\mathrm{C}$ ring (responsible for the uptake by erythrocytes) can act as intracellular substrates for PMOR. It is of note that the metabolites of quercetin enter erythrocytes and donate electrons to the PMOR as the parent compound. The present data show a relationship between the flavonoid structures and their ability to provide electrons to the PMOR, suggesting an additional mechanism whereby dietary flavonoids may exert beneficial effects in man.
\end{abstract}

Flavonoids: Trans-plasma membrane oxidoreductase: Ferricyanide: 1,1-Diphenyl-2-picryl-hydrazil

It is now well established that diets rich in fruits and vegetables are protective against the oxidative effects of reactive oxygen species, which are formed in vivo during the cellular aerobic metabolism and can cause damage to various cellular components such as DNA, proteins, lipids, etc (Steinmetz \& Potter, 1991a,b; Keli et al. 1996; Ness \& Powles, 1997; Ross \& Kasum, 2002). Despite the cells being well equipped with antioxidant defence systems, the accumulation of unrepaired products may be critical to the development of several important pathologies.

Flavonoids are polyphenol compounds, widely distributed in plant foods, which may exert beneficial effects in various diseases, including cancer, CVD and neurodegenerative disorders (Steinmetz \& Potter, 1991a,b; Richter et al. 1999). Many of the biological actions of flavonoids have been attributed to their antioxidant properties (Afanas'ev et al. 1989; Bors et al. 1990; Rice-Evans et al. 1997); more recently, it has been proposed that flavonoids and their metabolites may exert their effects by displaying modulatory actions in cells (Williams et al. 2004).

We have previously reported that flavonoids quercetin and myricetin are efficiently taken up by human erythrocytes and can act as substrates for the plasma membrane oxidoreductase (PMOR) activity, suggesting another mechanism whereby flavonoids can exert their protective effects (Fiorani et al. 2002). Indeed, this enzyme activity represents an important means to defend the cells against extracellular oxidative stressors (May et al. 1996), and since the erythrocytes are constantly exposed to oxidative stress, it might have a major role in maintaining a reduced plasma environment (Kennett \& Kuchel, 2003).

The aim of the present study was to evaluate the ability of several flavonoids (Fig. 1), commonly present in fruits and vegetables (flavonols: quercetin, myricetin, morin, kaempferol, fisetin; flavanol: catechin; flavones: luteolin, apigenin, acacetin, rutin; flavanones: taxifolin and naringenin; isoflavone: genistein) and of two reported in vivo quercetin metabolites, isorhamnetin and tamarixetin (Spencer et al. 2003a), to interact with human erythrocyte plasma membrane and induce extracellular reduction of oxidants.

\section{Materials and methods}

\section{Materials}

Flavonoids, 1,10-phenanthroline, ethyl acetate and 1,1-diphenyl2-picryl-hydrazil (DPPH) were purchased from Sigma-Aldrich Chemie (Steinheim, Germany). Isorhamnetin and tamarixetin were purchased from Extrasynthese (Z.I. Lyon Nord, Geney, France).

$\mathrm{K}_{3} \mathrm{Fe}(\mathrm{CN})_{6}, \mathrm{FeCl}_{3}$, citric acid, $\mathrm{NaH}_{2} \mathrm{PO}_{4}$ and acetonitrile (HPLC grade) were Carlo Erba products (Milan, Italy). 


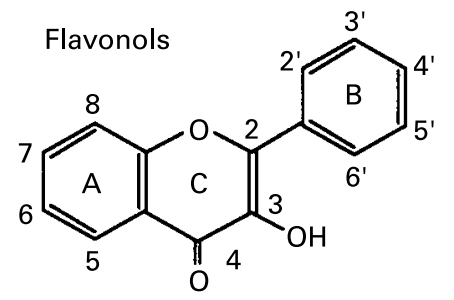

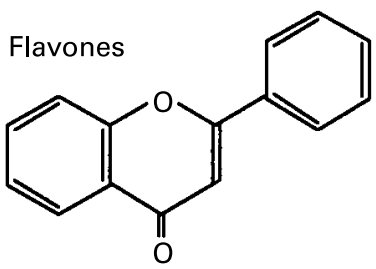<smiles>O=c1c(-c2ccccc2)coc2ccccc12</smiles>

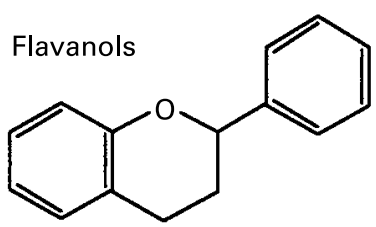<smiles>O=C1CC(c2ccccc2)Oc2ccccc21</smiles>

$\begin{array}{ll}\text { Quercitin } & 5,7,3^{\prime}, 4^{\prime}-\mathrm{OH} \\ \text { Myricetin } & 5,7,3^{\prime}, 4^{\prime}, 5^{\prime}-\mathrm{OH} \\ \text { Fisetin } & 3^{\prime}, 4^{\prime}, 7^{-}-\mathrm{OH} \\ \text { Kaempferol } & 5,7^{\prime} 4^{\prime}-\mathrm{OH} \\ \text { Morin } & 5,7^{\prime}, 5^{\prime}-\mathrm{OH} \\ \text { Isorhamnetin } & 5,7^{\prime} 4^{\prime}-\mathrm{OH}, 3^{\prime}-\mathrm{OCH}_{3} \\ \text { Tamarixetin } & 5,7^{\prime}, 3^{\prime}, \mathrm{OH}, 4^{\prime}-\mathrm{OCH}_{3}\end{array}$

Apigenin

Acacetin

Luteolin

$5,7,4^{\prime}-\mathrm{OH}$

$5,7,-\mathrm{OH}, 4^{\prime}-\mathrm{OCH}_{3}$

$5,7,3^{\prime}, 4^{\prime}-\mathrm{OH}$

$5,7,3^{\prime}, 4^{\prime}-\mathrm{OH}$

3-rhamnose

Genistein $\quad 5,7,4^{\prime}-\mathrm{OH}$

Catechin

$3,5,7,3^{\prime}, 4^{\prime}-\mathrm{OH}$

Taxifolin

$3,5,7,3^{\prime}, 4^{\prime}-\mathrm{OH}$

Naringenin

$5,7,4^{\prime}-\mathrm{OH}$

Fig. 1. Structures of the flavonoids.

\section{Methods}

Measurement of chemical ferricyanide reduction by different flavonoids. To a $1 \mathrm{~mm}$-ferricyanide (FIC) solution in PBS ( $\mathrm{pH}$ 7.4) were added different amounts of the flavonoid solution (final concentrations $2.5-500 \mu \mathrm{M}$ ). After standing for $30 \mathrm{~min}$ at $37^{\circ} \mathrm{C}$ (a time long enough to reach the reaction thermodynamic equilibrium), the ferrocyanide (FOC) formation was measured as reported by Avron \& Shavit (1963), using 1,10-phenanthroline as an indicator and measuring absorption at $510 \mathrm{~nm}(\varepsilon=10500 /$ M per $\mathrm{cm}$ ). The percentage of FOC formation (\% of reduction of the $1 \mathrm{~mm}$-FIC solution) was plotted against the sample concentration to obtain the $\mathrm{EC}_{50}$, defined as the flavonoid concentration required to obtain $50 \%$ of the maximal FIC-reducing activity.

Measurement of scavenging activity on 1,1-diphenyl-2-picrylhydrazil. The free radical-scavenging activity of flavonoids against the DPPH free radical was measured using a modified version of the method of Mallors \& Tappel (1966). Briefly, to $0.850 \mathrm{ml}$ of $100 \mu \mathrm{M}$-DPPH ethanolic solution were added different amounts of flavonoid solutions in ethanol (final concentration $1-150 \mu \mathrm{M}$ ) in $1 \mathrm{ml}$ cuvettes. After standing in the dark for $10 \mathrm{~min}$ (a time long enough to reach the reaction thermodynamic equilibrium), the absorbance (Abs) at $517 \mathrm{~nm}$ was measured. Controls containing ethanol instead of the flavonoid solution and blanks containing ethanol instead of DPPH solution were also made. The DPPH-scavenging activity was calculated according to the following formula: DPPH scavenging activity $(\%)=$ $\left(\mathrm{Abs}_{\mathrm{control}}-\mathrm{Abs}_{\text {sample }}\right) / \mathrm{Abs}_{\text {control }} \times 100$. The percentage of scavenging activity was plotted against the sample concentrations to obtain the $\mathrm{EC}_{50}$, defined as the flavonoid concentration required to obtain $50 \%$ of the maximal scavenging activity.

Human erythrocytes. Human venous blood (in heparin) from healthy volunteers was obtained by venepuncture. The erythrocytes were used immediately after sampling. The blood was centrifuged at $1861.5 \mathrm{~g}$ for $10 \mathrm{~min}$ at $4^{\circ} \mathrm{C}$. After removal of plasma, buffy coat, and the upper $15 \%$ of the packed erythrocytes, the erythrocytes were washed twice with cold PBS $(150 \mathrm{~mm}-\mathrm{NaCl}$, $5 \mathrm{mM}-\mathrm{Na}_{2} \mathrm{HPO}_{4}$, in deionised water, adjusted to $\left.\mathrm{pH} 7.4\right)$ and then re-suspended as described below.

Incubation of human erythrocytes with flavonoids. A stock solution $(20 \mathrm{mM})$ of each flavonoid was prepared in dimethyl 
sulfoxide and then diluted 1:2 with PBS. Packed erythrocytes $(10 \%, \mathrm{v} / \mathrm{v})$ were incubated in $\mathrm{PBS}$ at $37^{\circ} \mathrm{C}$ for $10 \mathrm{~min}$ in the presence of the flavonoids (flavonols: quercetin, myricetin, morin, kaempferol, fisetin; flavanol: catechin; flavones: luteolin, apigenin, acacetin, rutin; flavanones: taxifolin and naringenin; isoflavone: genistein) and of two reported in vivo quercetin metabolites, isorhamnetin and tamarixetin. After this time, the suspensions were immediately centrifuged at $1861.5 \mathrm{~g}$, the erythrocytes were washed twice with at least $50 \mathrm{vol}$. of PBS and then processed as reported below.

Measurement of ferricyanide reduction by human erythrocytes. FIC reduction was estimated as reported by Avron \& Shavit (1963). After exposure to the flavonoid, erythrocytes were washed twice with PBS and re-suspended (10\%, v/v) in PBS $+2.5 \mathrm{~mm}$-adenosine containing $1 \mathrm{~mm}$-FIC (potassium salt), dissolved immediately before use. The suspensions were incubated for $30 \mathrm{~min}$ at $37^{\circ} \mathrm{C}$ and then centrifuged at $1861.5 \mathrm{~g}$ at $4^{\circ} \mathrm{C}$. The resulting supernatant fractions were assayed for their FOC content using 1,10-phenanthroline as an indicator and measuring absorption at $510 \mathrm{~nm}(\varepsilon=10500 / \mathrm{M}$ per $\mathrm{cm})$.

Extracellular and intracellular content of flavonoids. The extracellular and intracellular content of flavonoids was measured as described by Ferrali et al. (1997), with slight modifications, by performing ethyl acetate extractions. To measure the extracellular concentration of flavonoids, the supernatant fraction obtained at the end of the incubation time was extracted three times with ethyl acetate. For the assessment of intracellular flavonoid content the erythrocyte lysates were extracted three times with ethyl acetate. The absorbance of the clear upper phase was measured spectrophotometrically at the wavelength corresponding to the maximal absorption spectrum (morin $380 \mathrm{~nm}$; quercetin, myricetin, isorhamnetin, tamarixetin, kaempferol $378 \mathrm{~nm}$; fisetin $370 \mathrm{~nm}$; rutin $340 \mathrm{~nm}$; apigenin $350 \mathrm{~nm}$; luteolin $336 \mathrm{~nm}$; acacetin, genistein $330 \mathrm{~nm}$; taxifolin $325 \mathrm{~nm}$; naringenin $320 \mathrm{~nm}$; catechin $285 \mathrm{~nm}$ ). The extra- and intracellular concentrations of the flavonoids were obtained from the corresponding calibration curve, performed in ethyl acetate (morin, $\varepsilon_{380}=8300 / \mathrm{M}$ per $\mathrm{cm}$; quercetin, $\varepsilon_{378}=26000 / \mathrm{M}$ per $\mathrm{cm}$; myricetin, $\varepsilon_{378}=18900 / \mathrm{M}$ per $\mathrm{cm}$; isorhamnetin, $\varepsilon \varepsilon_{378}=48700 / \mathrm{M}$ per $\mathrm{cm}$; tamarixetin, $\varepsilon_{378}=39300 / \mathrm{M}$ per $\mathrm{cm}$; kaempferol, $\varepsilon_{378}=22500 / \mathrm{M}$ per $\mathrm{cm}$; fisetin, $\varepsilon_{370}=22700 / \mathrm{M}$ per $\mathrm{cm}$; apigenin, $\varepsilon_{350}=15200 / \mathrm{M}$ per $\mathrm{cm}$; luteolin, $\varepsilon_{336}=68900 / \mathrm{M}$ per $\mathrm{cm}$; acacetin, $\varepsilon_{330}=16600 / \mathrm{M}$ per $\mathrm{cm}$; genistein, $\varepsilon_{330}=2500 / \mathrm{M}$ per $\mathrm{cm}$; taxifolin, $\varepsilon_{325}=3200 / \mathrm{M}$ per $\mathrm{cm}$; naringenin, $\varepsilon_{320}=2200 / \mathrm{M}$ per $\mathrm{cm}$; catechin $\varepsilon_{285}=4 \cdot 700 / \mathrm{M}$ per $\mathrm{cm}$ ). In all experiments, ethyl acetate extraction of a control sample was performed (either erythrocyte or supernatant fraction), in order to look for possible interferences with erythrocyte constituents. The results obtained showed that the absorbance values of the erythrocyte control samples were identical to those of an ethyl acetate solution.

The percentage of haemolysis was evaluated in the same samples by measuring the $\mathrm{Hb}$ concentration $v$. the total $\mathrm{Hb}$ content. The extent of lysis was not different from controls and never higher than $0.5 \%$.

High-performance liquid chromatography analysis of quercetin and its methylated metabolites. After incubation of the human erythrocyte suspensions with $50 \mu \mathrm{M}$-quercetin, tamarixetin or isorhamnetin as described earlier, the samples were centrifuged at $1861.5 \mathrm{~g}$ for $5 \mathrm{~min}$ and the supernatant fractions were collected. The packed erythrocytes were then extensively washed with PBS and lysed with cold bi-distilled water. Both samples (extracellular milieu and erythrocyte lysate) were extracted three times with ethyl acetate. All the samples were taken to dryness by rotary evaporation and re-dissolved in dimethyl sulfoxide and diluted with bi-distilled water just before HPLC analysis. HPLC analysis of quercetin and its methylated derivatives was performed by using a $25 \times 4.6 \mathrm{~mm}$ Discovery C18 $(5 \mu \mathrm{m}$; Supelco, Bellefonte, PA, USA) equipped with a Supelguard Discovery C-18 guard column $(2 \mathrm{~cm} \times 4 \mathrm{~mm}, 5 \mu \mathrm{m})$. A modified version of the analytical HPLC method from Day et al. (2000) was used. Solvent A ( $0.1 \%$ trifluoroacetic acid) and B (acetonitrile) were run at a flow rate of $1 \mathrm{ml} / \mathrm{min}$. The running gradient was adjusted to $17 \% \mathrm{~B}$ ( $2 \mathrm{~min})$, increasing to $25 \% \mathrm{~B}$ ( $5 \mathrm{~min}), 35 \%$ $\mathrm{B}(8 \mathrm{~min}), 50 \% \mathrm{~B}(5 \mathrm{~min})$ and then $100 \% \mathrm{~B}(10 \mathrm{~min})$, followed by a re-equilibration at $17 \% \mathrm{~B}(15 \mathrm{~min})$. The injection volume was $100 \mu \mathrm{l}$ and the eluate was monitored at $270 \mathrm{~nm}$.

\section{Results and discussion}

\section{In vitro ferricyanide-reducing activity}

The direct interaction of the various flavonoids (belonging to the family of flavonol, flavanol, flavone, flavanone and isoflavone) with an oxidant was investigated by monitoring the formation of its reduction product. For this purpose, increasing concentrations $(2.5$ to $500 \mu \mathrm{M})$ of the flavonoids were incubated in PBS containing $1 \mathrm{~mm}$-FIC and FOC formation was detected spectrophotometrically after $30 \mathrm{~min}$. As reported in Fig. 2, quercetin and myricetin, followed by taxifolin, rutin and fisetin, displayed a high FIC-reducing activity.

These compounds are characterised by the presence of a B-ring catechol group (dihydroxylated B-ring) capable of readily donating hydrogen (i.e. electron). The substitution of $3^{\prime}-\mathrm{OH}$ or $4^{\prime}-\mathrm{OH}$ of quercetin with a methyl group, as in isorhamnetin or tamarixetin, respectively, only slightly decreased the FIC-reducing activity. A somewhat greater decrease was noticed by changing

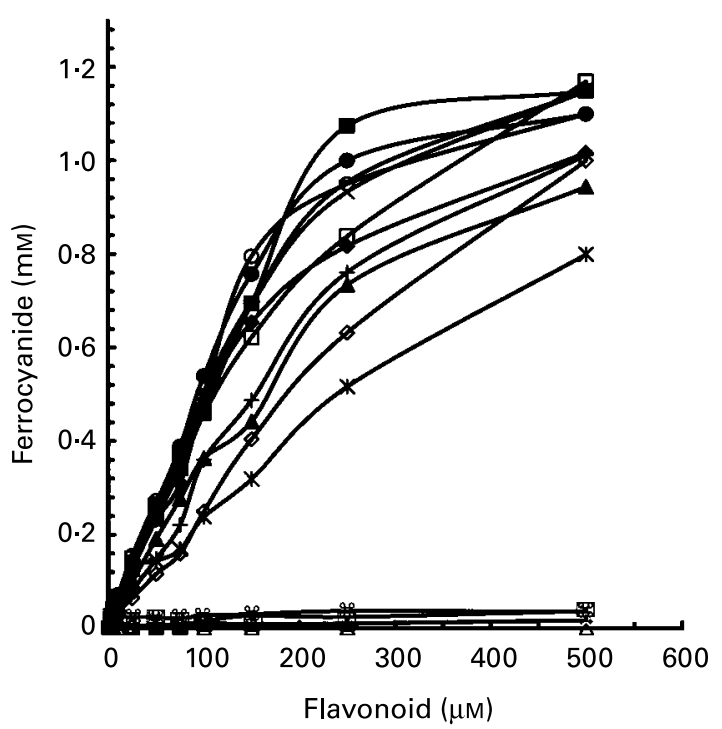

Fig. 2. Ferricyanide reduction by various flavonoids. Ferricyanide $(1 \mathrm{~mm})$ in

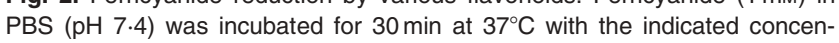
trations of quercetin $(\bigcirc)$, myricetin $(\bullet)$, fisetin $(\square)$, luteolin $(\boldsymbol{\Delta})$, isorhamnetin $(\square)$, taxifolin $(\bullet)$, kaempferol $(\times)$, tamarixetin $(\bullet)$, morin $(+)$, catechin $(*)$, rutin $(-)$, apigenin $(\triangle)$, acacetin $(\mathscr{H})$, genistein $(\bullet)$ and naringenin $(\otimes)$. Then, ferrocyanide formation was assayed (for details, see p. 339). 
the $3^{\prime}, 4^{\prime}$ orthodihydroxy arrangement of the $\mathrm{B}$ ring to $3^{\prime}, 5^{\prime}$ metadihydroxy, as in morin. Kaempferol, a flavonol identical to quercetin, but having no hydroxyl group at the $3^{\prime}$ position in the B ring, displayed an even lower activity. The 2,3 double bond in the $\mathrm{C}$ ring does not appear to play a major role for FIC-reducing activity, since taxifolin displayed remarkably similar effects to quercetin. However, catechin, which differs from taxifolin lacking the 4-oxo group in the $\mathrm{C}$ ring, showed a strong decrease in FICreducing activity. The substitution of 3-OH of quercetin with 3-Orutinoside, as in rutin, decreased the reducing activity by about $10 \%$, whereas a $50 \%$ reduction was observed upon dehydroxylation in the 3-position, as in luteolin. Finally, dehydroxylation at both the 3- and $3^{\prime}$-positions led to a complete loss of activity, as in apigenin, acacetin (4'-methoxyapigenin) and naringenin, the latter lacking 2,3 double bonds as well. The isoflavone genistein was also inactive.

Table 1 reports the $\mathrm{EC}_{50}$ values as calculated from the curves illustrated in Fig. 2.

Taken together, these results emphasise the relevance of the catechol-like structure of the $\mathrm{B}$ ring in association with the 3-OH group on the expression of maximal FIC-reducing activity, and show that the substitution of 3-OH with 3-O-rutinoside as in rutin, or the methylation of $3^{\prime}$ or $4^{\prime}-\mathrm{OH}$ as in isorhamnetin or tamarixetin only slightly reduce the antioxidant activity of these compounds.

It is of note that the very high reducing activities displayed by most flavonoids tested have also been observed by other authors. As reported by Cao et al. (1997) the flavonoids that contain multiple $\mathrm{OH}$ substitutions (i.e. myricetin, quercetin, luteolin, etc) showed peroxyl radical-scavenging activity several times higher than Trolox, an $\alpha$-tocopherol analogue. They reported that the stoichiometric factor (i.e. the number of peroxyl radicals trapped per molecule of antioxidant) of these flavonoids is about $6-9$, whereas the stoichiometric factor of Trolox is 2. Moreover, other authors (Firuzi et al. 2005) evaluated the antioxidant activities of flavonoids by 'ferric-reducing antioxidant power' measurement. They showed that quercetin, fisetin and myricetin appeared the most active compounds in the ferric-reducing antioxidant power assay and they were $3.02,2.52$ and 2.28 times more active than Trolox, respectively.

\section{1,1-Diphenyl-2-picryl-hydrazil scavenging activity}

As a further approach to estimate the relative potency of the tested flavonoids in reactions involving electron donation, we used the DPPH assay, widely employed for the assessment of antioxidant activity. DPPH, a stable organic free radical, displays a characteristic absorption maximum between 515 and $517 \mathrm{~nm}$ that diminishes in the presence of compounds reducing it to its hydrazine form by hydrogen-electron donation. The different kinetic behaviour of antioxidants is an important factor in the evaluation of the radical-scavenging activity (Bandonienè \& Murkovic, 2002). Fig. 3 (EC 50 values are shown in Table 1) illustrates the scavenging activity of the tested flavonoids after a $10 \mathrm{~min}$ incubation in ethanol in the presence of $\mathrm{DPPH}^{-}(100 \mu \mathrm{M})$. Complete conversion of $\mathrm{DPPH}$ to its hydrazine derivative was observed at much lower concentrations of the flavonoids than those necessary to fully reduce FIC, an event probably ascribable to the different concentration of the oxidising substrate employed in the two experimental conditions. The results obtained were nevertheless similar to those previously described for the FICreduction assay, with some important exceptions. Quercetin, myricetin and fisetin most effectively scavenged the DPPH radical, confirming previous results showing that the scavenging activity of flavonoids is related to the presence of (i) a $3^{\prime}-4^{\prime}$ dihydroxylated $\mathrm{B}$ ring, (ii) the 2,3-double bond in conjugation with a 4oxo function in the $\mathrm{C}$ ring and finally (iii) the additional presence of both 3- and 5- hydroxyl groups (Williams et al. 2004). It should be noted, however, that in contrast with the results obtained measuring FIC reduction, the substitution of $3^{\prime}$ or $4^{\prime}-$ $\mathrm{OH}$ with a methyl group (for example, isorhamnetin or tamarixetin) or 3-OH with 3-O-rutinoside (for example, rutin) substantially reduced the scavenging activity. The remaining compounds, either inactive or poorly active in the FIC assay, produced similar outcomes in the DPPH assay.

\section{Human erythrocyte ferricyanide-reducing activity promoted by different flavonoids}

FIC is a mild oxidant that does not cross the cell membrane and accepts electrons from the PMOR activity (Himmelreich \& Kuchel, 1997; van Duijn et al. 1998). Although the physiological electron donors for this enzyme are ascorbic acid and NADH (May, 1999; May et al. 1999), our recent findings indicate that quercetin and myricetin are also potent electron donors for the PMOR in human erythrocytes (Fiorani et al. 2002, 2003). Experiments were therefore performed to determine the potency of the tested flavonoids in supporting the ability of human

Table 1. Flavonoid concentrations required to obtain $50 \%$ of the maximal effect (EC $\mathrm{C}_{50}$ values) concerning ferricyanide reduction and 1,1-diphenyl-2-picryl-hydrazil (DPPH)-scavenging activity* $^{*}$

\begin{tabular}{|c|c|c|}
\hline Flavonoid & $\mathrm{EC}_{50}$ ferricyanide reduction $(\mu \mathrm{m})$ & $\mathrm{EC}_{50} \mathrm{DPPH}$-scavenging activity $(\mu \mathrm{m})$ \\
\hline Quercetin & $96 \cdot 5$ & $6 \cdot 23$ \\
\hline Myricetin & $97 \cdot 3$ & $6 \cdot 37$ \\
\hline Fisetin & $114 \cdot 8$ & $6 \cdot 75$ \\
\hline Rutin & $116 \cdot 1$ & $9 \cdot 67$ \\
\hline Luteolin & $160 \cdot 0$ & $12 \cdot 79$ \\
\hline Isorhamnetin & $108 \cdot 0$ & 12.94 \\
\hline Taxifolin & $118 \cdot 3$ & $13 \cdot 83$ \\
\hline Kaempferol & $197 \cdot 1$ & $18 \cdot 4$ \\
\hline Catechin & $282 \cdot 6$ & $18 \cdot 55$ \\
\hline Tamarixetin & $112 \cdot 0$ & $18 \cdot 56$ \\
\hline Morin & $159 \cdot 4$ & $27 \cdot 23$ \\
\hline
\end{tabular}

* The values have been calculated from the curves reported in Figs. 2 and 3 respectively. 


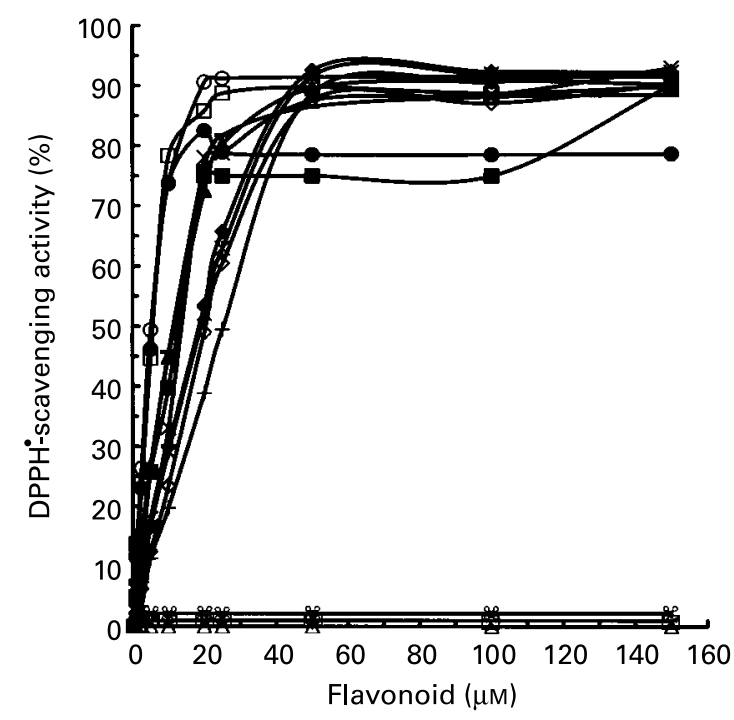

Fig. 3. 1,1-Diphenyl-2-picryl-hydrazil (DPPH)-scavenging activity by various flavonoids. DPPH $(100 \mu \mathrm{M})$ in ethanol was incubated for $10 \mathrm{~min}$ at $37^{\circ} \mathrm{C}$ with the indicated concentrations of quercetin $(\bigcirc)$, myricetin $(\bullet)$, fisetin $(\square)$, luteolin $(\boldsymbol{\Delta})$, isorhamnetin $(\boldsymbol{\square})$, taxifolin $(\times)$, kaempferol $(\diamond)$, tamarixetin $(\diamond)$, morin $(+)$, catechin $(*)$, rutin $(-)$, apigenin $(\triangle)$, acacetin $(\mathscr{H})$, genistein $(\bullet)$ and naringenin $(\otimes)$ dissolved in ethanol. The decrease of the absorbance at $517 \mathrm{~nm}$ was then measured and the scavenging activity was expressed as percentage of the absorbance of the control DPPH solution.

erythrocytes to reduce extracellular oxidants. For this purpose, erythrocytes were first incubated for $10 \mathrm{~min}$ with a $50 \mu \mathrm{M}$ concentration of each flavonoid, centrifuged and extensively rinsed, resuspended in PBS supplemented with adenosine and $1 \mathrm{mM}$-FIC and finally incubated for $30 \mathrm{~min}$ at $37^{\circ} \mathrm{C}$. Formation of $\mathrm{FOC}$ was subsequently measured in the supernatant fraction. It is important to note that this response, as previously shown for quercetin (Fiorani et al. 2002), is mediated by the activity of the PMOR and not by the flavonoids released from the cells. For this purpose, erythrocytes were incubated with $50 \mu \mathrm{M}$-flavonoids (listed earlier; p. 339) for $10 \mathrm{~min}$, washed twice with at least 50 vol. of PBS and then incubated (10\%, v/v) with PBS plus adenosine. After $30 \mathrm{~min}$, the erythrocyte suspensions were centrifuged and flavonoid content was assayed either in the supernatant fractions and in erythrocytes after ethyl acetate extractions, as detailed earlier (p. 340). The results obtained consistently demonstrated that none of the flavonoids was significantly released during post-treatment incubations (not shown; Fiorani et al. 2002). As illustrated in Fig. 4, quercetin and myricetin, followed by fisetin, isorhamnetin and tamarixetin were the most potent compounds in promoting the PMOR activity. Kaempferol, luteolin, taxifolin and morin were significantly less effective and the remaining flavonoids were inactive. For a correct interpretation of these results the cellular uptake of the different flavonoids was next assessed under equal loading conditions. Fig. 5 shows that quercetin, myricetin, kaempferol, fisetin, morin, luteolin, isorhamnetin, tamarixetin, apigenin and acacetin, were efficiently taken up by the erythrocytes and actually accumulated at levels above the initial extracellular loading concentration. The uptake of naringenin, genistein, taxifolin and finally catechin was remarkably lower and that of rutin was virtually undetectable.

Thus, all compounds displaying significant FIC-reducing activity (Fig. 2), and efficiently taken up by the erythrocytes

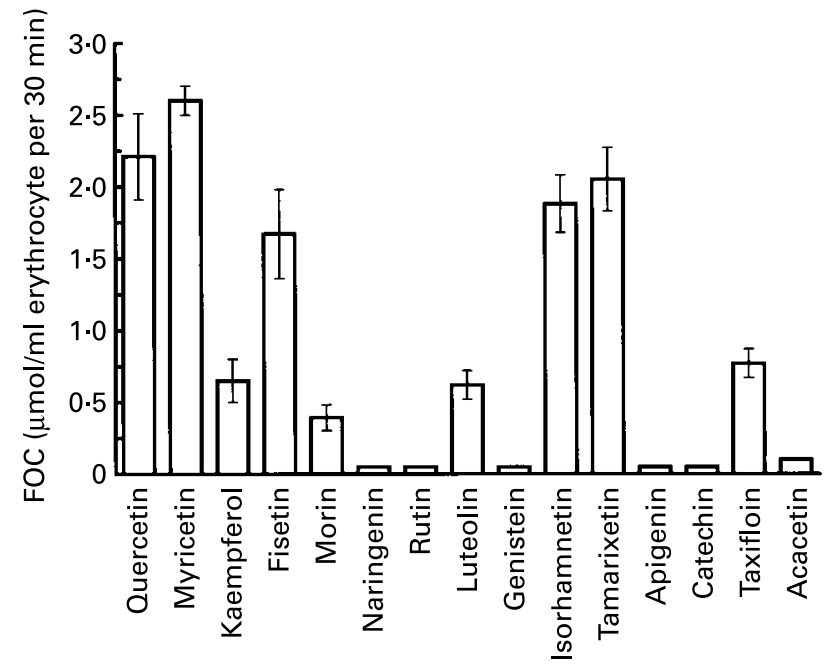

Fig. 4. Effect of various flavonoids on the rate of ferricyanide (FIC) reduction in human erythrocytes. Human packed erythrocytes were incubated for $10 \mathrm{~min}$ at $37^{\circ} \mathrm{C}$ in PBS $(10 \%, \mathrm{v} / \mathrm{v})$ in the presence of $50 \mu \mathrm{M}$-flavonoid. After centrifugation the cells were washed twice with PBS and the packed erythrocytes were re-suspended in PBS $(10 \%, \mathrm{v} / \mathrm{v})$ plus $2.5 \mathrm{~mm}$-adenosine containing $1 \mathrm{~mm}-\mathrm{FIC}$. After $30 \mathrm{~min}$ of incubation at $37^{\circ} \mathrm{C}$, the cell suspensions were centrifuged and the ferrocyanide (FOC) content was assayed as detailed on p. 340. Flavonoid-dependent FIC-reducing activity was determined upon subtraction of basal FIC-reducing activity detected in the untreated samples. All data are the means of at least three independent determinations, with standard deviations represented by vertical bars.

(Fig. 5), act in parallel as intracellular substrates for the PMOR activity. Examples are quercetin, myricetin, fisetin as well as the in vivo plasma metabolites of quercetin, isorhamnetin and tamarixetin. It is important to point out that the two latter compounds were not converted within the erythrocytes to their de-

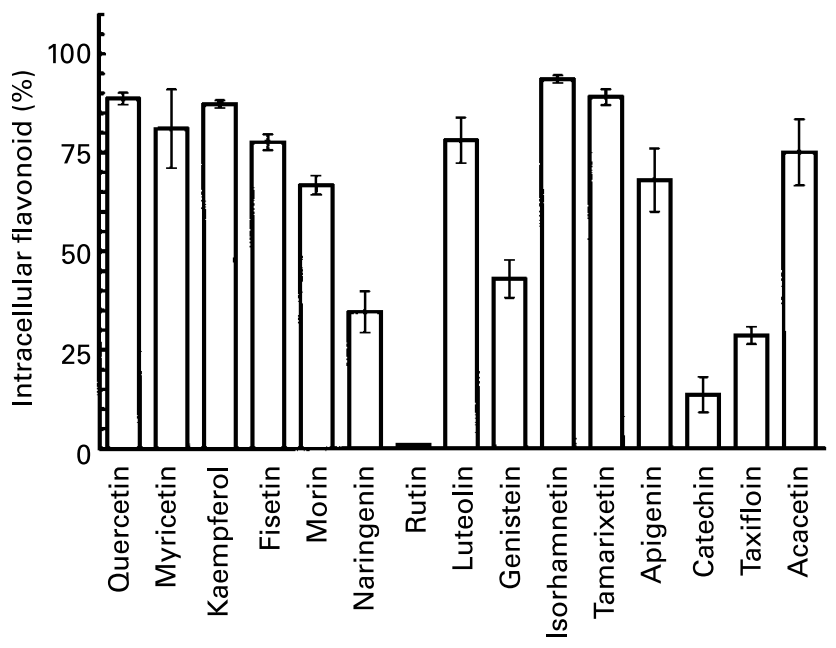

Fig. 5. Flavonoid uptake by human erythrocytes. Human packed erythrocytes were incubated for $10 \mathrm{~min}$ at $37^{\circ} \mathrm{C}$ in PBS $(10 \%, \mathrm{v} / \mathrm{v})$ in presence of $50 \mu \mathrm{M}$ flavonoid. After centrifugation, the cells were washed twice with at least $50 \mathrm{vol}$. of PBS, then lysed by re-suspending with 3 vol. of cold distilled water and maintained $10 \mathrm{~min}$ at $4^{\circ} \mathrm{C}$. Flavonoids were extracted from either the haemolysate or supernatant fraction with ethyl acetate, as described on p. 340. All data are the means of at least three independent determinations, with standard deviations represented by vertical bars. The data are expressed as percentage of the flavonoid recovered in erythrocytes with regard to the total flavonoid content (extra + intracellular concentration). 

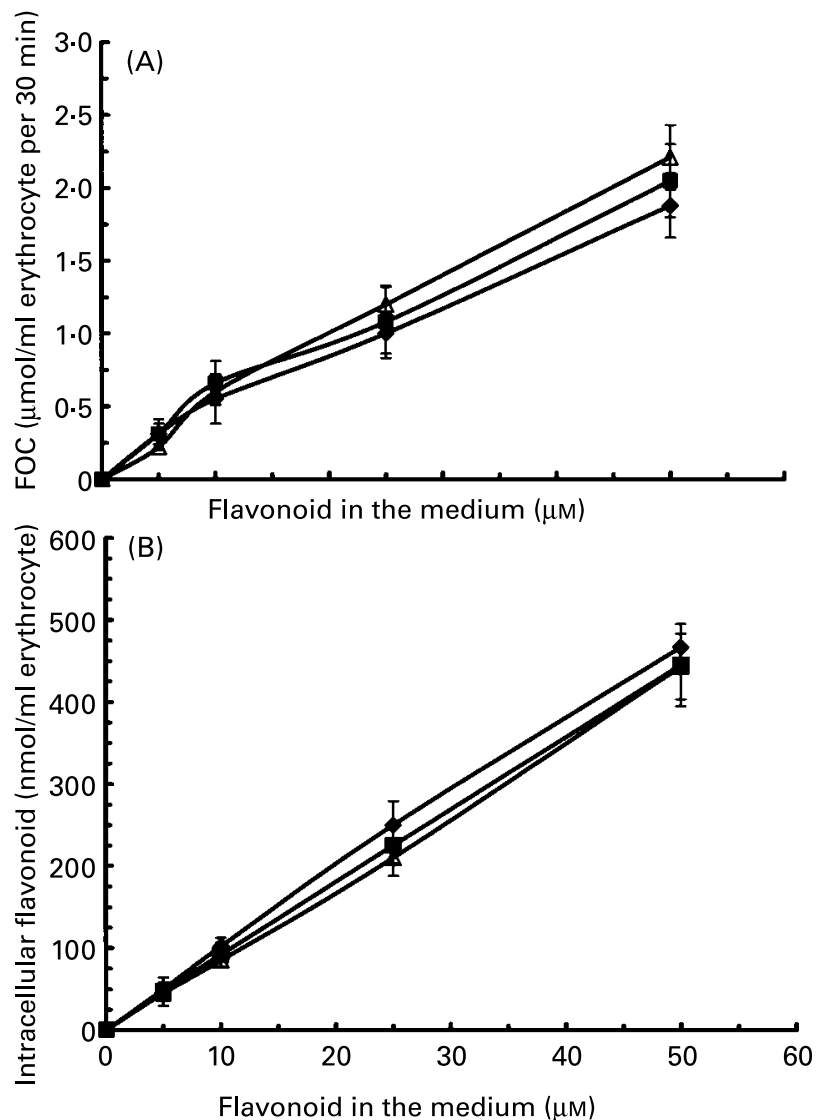

Fig. 6. Ferricyanide (FIC)-reducing activity (A) and flavonoid uptake (B) by human erythrocytes. Human packed erythrocytes were incubated for $10 \mathrm{~min}$ at $37^{\circ} \mathrm{C}$ in PBS $(10 \%, v / v)$ with $50 \mu \mathrm{M}$-flavonoid (isorhamnetin (ם); tamarixetin $(\diamond)$; quercetin $(\triangle))$. After centrifugation the cells were washed twice with PBS and the packed erythrocytes were: (A) re-suspended at $10 \%$ packed cell volume in PBS plus $2.5 \mathrm{~mm}$-adenosine containing $1 \mathrm{~mm}-\mathrm{FIC}$ and the ferrocyanide $(F O C)$ formation was assayed as reported in the legend of Fig. 4, or (B) lysed by re-suspending with 3 vol. of cold distilled water and the flavonoid content was measured as described in the legend of Fig. 5. Flavonoiddependent FIC-reducing activity was determined upon subtraction of basal FIC-reducing activity detected in the untreated samples. All data are the means of at least three independent determinations, with standard deviations represented by vertical bars.

methylated form (for example, to quercetin), an event that would explain the identical abilities to reduce extracellular oxidants mediated by the PMOR activity. This notion is clearly established by the HPLC analysis, which revealed that only isorhamnetin or tamarixetin are recovered inside the cell after exposure of erythrocytes to the methylated derivatives.

Fig. 6 (A) shows concentration-dependence curves of the FICreducing activity of isorhamnetin, tamarixetin and quercetin by human erythrocytes. As illustrated, the two methylated metabolites are able to act as intracellular substrates of the PMOR already at concentrations $\leq 5 \mu \mathrm{M}$ with a rate quite similar to that of quercetin. Similarly, the intracellular uptake of these compounds was substantially identical (Fig. 6 (B)).

Additional flavonoids, namely kaempferol, morin and luteolin, were also efficiently taken up by the erythrocytes (Fig. 5) but displayed poor direct (Fig. 2) or cell-mediated (Fig. 4) FIC reduction. Taxifolin was less efficiently taken up by the erythrocytes (Fig. 5) and, while more effective than kaempferol and luteolin in reducing FIC (Fig. 2), caused a similar erythrocytedependent reduction of extracellular oxidants (Fig. 4). Although apigenin, acacetin, genistein and naringenin were accumulated in the erythrocytes in fairly high amounts (Fig. 5), these compounds were unable to reduce FIC chemically (Fig. 2) and in the cell-mediated assay (Fig. 4). Rutin was similar to quercetin in promoting chemical reduction of FIC (Fig. 2) but was not taken up by the erythrocytes (Fig. 5) and therefore unable to behave as intracellular substrates for the PMOR activity (Fig. 4). Finally, catechin did not permeate the cell membrane (Fig. 5) and thus was inactive in the cell-mediated assay (Fig. 4), while it was poorly effective in the chemical FIC-reduction assay (Fig. 2). It is of note that taxifolin, which shows a chemical FIC-reducing activity similar to that of quercetin (Fig. 2), contributes to a lesser extent to the PMOR activity (Fig. 4). This fact is quite probably linked to the poor uptake of taxifolin by human erythrocytes (Fig. 5). This flavonoid, as already mentioned, differs from quercetin at the 2,3 position, lacking in the double bond. Naringenin, which, as taxifolin, lacks a 2,3 double bond, penetrates the erythrocyte membrane with a percentage similar to taxifolin. However, naringenin does not display chemical FIC-reduction activity or DPPH-scavenging ability. This fact is probably linked to the lack of 3 - and $3^{\prime}$-hydroxyl groups. Catechin, which does not have either the 2,3 double bond or a 4-carbonyl group, penetrates the erythrocyte membrane in very small amounts.

Therefore, it could be attributed to the 2,3 double bond and to the 4-carbonyl group ring a pivotal role on flavonoid uptake by erythrocytes.

Kaempferol and morin, although efficiently taken up by erythrocytes, were remarkably less effective than quercetin in promoting reduction of extracellular FIC. This observation confirms the previous results, emphasising the notion that the cathecol plays a pivotal role in the biological response mentioned earlier.

In conclusion, in the present study the in vitro evidence of antioxidant capacity of various polyphenolic compounds was related with that obtained from a biological system represented by human erythrocytes. The results obtained show that the flavonoids, which possess the catechol structure in the $\mathrm{B}$ ring (responsible for the reducing activity), in conjunction with a 2,3 double bond and 4-oxo function in the $\mathrm{C}$ ring (which favour the uptake of flavonoid by erythrocytes) appear to be the most potent agents in acting as electron donors to the PMOR activity. On the other hand, the role of quinone and quinone methide should be also considered, oxidation products of quercetin (Awad et al. 2000, 2001), which may display cytotoxic effects (MacGregor \& Jurd, 1978; Sahu \& Washington, 1991; Bolton et al. 1998; Penning et al. 1999). However, according to the literature (Cao et al. 1997; Lee et al. 2003) the pro-oxidant activities of natural antioxidants are unlikely to be a significant problem 'in vivo', and most of the studies show that flavonoids exert beneficial effects against pathological conditions such as CVD, cancer (Steinmetz \& Potter, 1991 a,b) and neurodegenerative disorders. Therefore, diets rich in these phenolic compounds are now strongly recommended. However, some doubts on the physiological relevance of these results arise from the bioavailability of these compounds. In fact, it should be underlined that plasma concentrations reached after flavonoid consumption vary highly according to the nature of the polyphenol and to the food source. Data reported in the literature show that the plasma concentrations of total metabolites can vary from 0 to $4 \mu \mathrm{mol} / \mathrm{l}$ after consumption of $50 \mathrm{mg}$ aglycone equivalents, and the relative urinary excretion ranged from 0.3 to $43 \%$ of the ingested dose, depending on polyphenolic compounds (Manach et al. 2005). 
Moreover, as pointed out by Kroon et al. (2004), most of the dietary polyphenols undergo extensive modifications during transfer across the small intestine and then again in the liver, so that the forms reaching the blood and tissues are in general neither aglycones (except for green tea catechins) nor the various glycosides as the dietary source (Kroon et al. 2004). Circulating glucuronides, sulfates, and $O$-methylated forms are believed to be those most likely to exert bioactivity and express beneficial effects in human subjects and animals (Spencer et al. 2001a,b, 2003b, 2004; Guglielmone et al. 2002; Schroeter et al. 2003). It should be considered, however, as pointed out by Spencer et al. (2004), that there is the possibility that both flavonoid and $O$ methylated flavonoid glucuronides may be de-conjugated by the action of $\beta$-glucuronidases present in human tissues such as liver or small intestine or during local conditions of inflammation. In this case, free aglycone or $O$-methylated forms will be released and may go on to express cellular effects. Indeed, glucuronidases are present in a number of tissues within the body (Tukey et al. 2000) and may be released by certain cells.

In the bloodstream, erythrocytes encounter a variety of oxidant stressors which can be both endogenous, from cellular generation of superoxide and $\mathrm{H}_{2} \mathrm{O}_{2}$ (van Dyke \& Saltman, 1996) and exogenous in areas of inflammation. Therefore, the PMOR activity in erythrocytes can play a protective role by reducing extracellular oxidants. The fact that specific flavonoids, abundantly present in fruits and vegetables as well as their plasma metabolites, act as substrates for this membrane enzyme provides an efficient way to maintain a redox state in the plasma, supporting the statement of the beneficial effects of dietary flavonoids.

\section{Acknowledgements}

We would like to thank the staff of the blood transfusion centre of Urbino Hospital for kindly providing blood samples from human volunteers.

\section{References}

Afanas'ev IB, Dorozhko AI, Brodskii AW, Korstyuk VA \& Potapovich AI (1989) Chelating and free radical scavenging mechanisms of inhibitory action of rutin and quercetin in lipid peroxidation. Biochem Pharmacol 38, 1763-1768.

Avron M \& Shavit N (1963) A sensitive simple method for determination of ferrocyanide. Anal Biochem 6, 549-555.

Awad HM, Boersma MG, Boeren S, van Bladeren J, Vervoort J \& Rietjens IMCM (2001) Structure-activity study on quinone/quinone methide chemistry of flavonoids. Chem Res Toxicol 14, 398-408.

Awad HM, Boersma MG, Vervoort J \& Rietjens IMCM (2000) Peroxidase-catalyzed formation of quercetin quinine methide-glutathione adducts. Arch Biochem Biophys 378, 224-233.

Bandonienè D \& Murkovic M (2002) On line HPLC-DPPH screening method for evaluation of radical scavenging phenols extracted from apples (Malus domestica L.). J Agric Food Chem 50, 2482-2487.

Bolton JL, Pisha E, Zhang F \& Qiu S (1998) Role of quinoids in estrogen carcinogenesis. Chem Res Toxicol 11, 1113-1126.

Bors W, Heller W, Michel C \& Saran M (1990) Flavonoids as antioxidants: determinations of radical scavenging efficiences. Methods Enzymol 234, 343-355.

Cao G, Sofic E \& Prior R (1997) Antioxidant and prooxidant behaviour of flavonoids: structure-activity relationship. Free Rad Biol Med 22, $749-760$.
Day AJ, Bao Y, Morgan MRA \& Williamson G (2000) Conjugation position of quercetin glucuronides and effect on biological activity. Free Rad Biol Med 29, 1234-1243.

Ferrali M, Signorini C, Caciotti B, Sugherini L, Ciccoli L, Giacchetti D \& Comporti M (1997) Protection against oxidative damage of erythrocyte membrane by the flavonoid quercetin and its relation to iron chelating activity. FEBS Lett 416, 123-129.

Fiorani M, Accorsi A \& Cantoni O (2003) Human red blood cells as a natural flavonoid reservoir. Free Rad Res 37, 1331-1338.

Fiorani M, De Sanctis R, De Bellis R \& Dachà M (2002) Intracellular flavonoid as electron donors for extracellular ferricyanide reduction in human erythrocytes. Free Rad Biol Med 32, 64-72.

Firuzi O, Lacanna A, Petrucci R, Marrosu G \& Saso L (2005) Evaluation of the antioxidant activity of flavonoids by 'ferric reducing antioxidant power' assay and cyclic voltametry. Biochem Biophys Acta 1721, 174-184.

Guglielmone HA, Agnese AM, Nunez Montoya SC \& Cabrera JL (2002) Anticoagulant effect and action mechanism of sulphated flavonoids from Flaveria bidentis. Thromb Res 105, 183-188.

Himmelreich U \& Kuchel PW (1997) ${ }^{13} \mathrm{C}$-NMR studies of transmembrane electron transfer to extracellular ferricyanide in human erythrocytes. Eur J Biochem 246, 638-645.

Keli SO, Hertog MG, Feskens EJ \& Kromhout D (1996) Dietary flavonoids, antioxidant vitamins, and incidence of stroke: the Zutphen study. Arch Intern Med 156, 637-642.

Kennett EC \& Kuchel PW (2003) Redox reaction and electron transfer across the red cell membrane. IUBMB Life 55, 375-385.

Kroon PA, Clifford MN, Day AJ, Donovan JL, Manach C \& Williamson G (2004) How should we assess the effects of exposure to dietary polyphenols in vitro? Am J Clin Nutr 80, 15-21.

Lee JC, Park JK, Chung GH \& Jang YS (2003) The antioxidant, rather than prooxidant, activities of quercetin on normal cells: quercetin protects mouse thymocytes from glucose oxidase-mediated apoptosis. Exp Cell Res 291, 386-397.

MacGregor JT \& Jurd L (1978) Mutagenicity of plant flavonoids: structural requirements for mutagenic activity in Salmonella typhimurium. Mutat Res 54, 297-309.

Mallors A \& Tappel AL (1966) The inhibition of mitochondrial peroxidation by ubiquinone and ubiquitol. J Biol Chem 241, 4353-4356.

Manach C, Williamson G, Morand C, Scalbart A \& Remesy C (2005) Bioavailability and bioefficacy of polyphenols in humans. I. Review of 97 bioavailability studies. Am J Clin Nutr 21, 230S-242S.

May JM (1999) Is ascorbic acid an antioxidant for the plasma membrane? FASEB J 13, 995-1006.

May JM, Mendiratta S, Qu ZC \& Loggins E (1999) Vitamin C recycling and function in human monocytes U-937 cells. Free Rad Biol Med 26, $1513-1523$.

May JM, Qu ZC \& Morrow JD (1996) Interaction of ascorbate and ( $\alpha$-tocopherol in resealed human erythrocyte ghosts. J Biol Chem 271, 10577-10582.

Ness AR \& Powles JW (1997) Fruits, vegetables and cardiovascular disease: a review. Int J Epidemiol 26, 1-13.

Penning TM, Burczynski ME, Hung CF, McCoull KD, Palackal NT \& Tsuruda LS (1999) Dihydrodiol dehydrogenases and polycyclic aromatic hydrocarbon activation: generation of reactive and redox active o-quinones. Chem Res Toxicol 12, 1-18.

Rice-Evans CA, Miller NJ \& Paganga G (1997) Antioxidant properties of phenolic compounds. Trends Plant Sci 2, 152-159.

Richter M, Ebermann R \& Marian B (1999) Quercetin-induced apoptosis in colorectal tumor cells: possible role of EGF receptor signaling. Nutr Cancer 34, 88-99.

Ross AJ \& Kasum CM (2002) Dietary flavonoids: bioavailability, metabolic effects, and safety. Annu Rev Nutr 22, 19-34.

Sahu SC \& Washington MC (1991) Quercetin-induced lipid peroxidation and DNA damage in isolated rat-liver nuclei. Cancer Lett 58, 75-79.

Schroeter H, Boyd CS, Ahmed R, Spencer JPE, Duncan RF, Rice-Evans C \& Cadenas E (2003) c-Jun N-terminal kinase (JNK)-mediated modulation 
of brain mitochondria function: new target proteins for JNK signalling in mitochondrion-dependent apoptosis. Biochem J 372, 359-369.

Spencer JPE, Abd-El-Mohsen MM \& Rice-Evans C (2004) Cellular uptake and metabolism of flavonoids and their metabolites: implication for their bioactivity. Arch Biochem Biophys 423, 148-161.

Spencer JPE, Kuhnle G, Williams RJ \& Rice-Evans C (2003a) Intracellular metabolism and bioactivity of quercetin and its in vivo metabolites. Biochem J 372, 173-181.

Spencer JPE, Rice-Evans C \& Williams RJ (2003b) Modulation of prosurvival Akt/protein kinase B and ERK1/2 signaling cascades by quercetin and its in vivo metabolites underlie their action on neuronal viability. J Biol Chem 278, 34783-34793.

Spencer JPE, Schroeter H, Crossthwaithe AJ, Kuhnle G, Williams RJ \& Rice-Evans C (2001a) Contrasting influences of glucuronidation and $O$-methylation of epicatechin on hydrogen peroxide-induced cell death in neurons and fibroblasts. Free Rad Biol Med 31, 1139-1146.

Spencer JPE, Schroeter H, Kuhnle G, Srai SK, Tyrrell RM, Hahn U \& Rice-Evans C (2001b) Epicatechin and its in vivo metabolite, 3'-O- methyl epicatechin, protect human fibroblasts from oxidative-stressinduced cell death involving caspase-3 activation. Biochem $J$ 345, 493-500.

Steinmetz KA \& Potter JD (1991a) Vegetables, fruit and cancer I. Epidemiology. Cancer Causes Control 5, 325-337.

Steinmetz KA \& Potter JD (1991b) Vegetables, fruit and cancer II. Mechanisms. Cancer Causes Control 5, 427-442.

Tukey RH \& Strassburg CP (2000) Human UDP-glucuronosyltransferases: metabolism, expression, and disease. Аnпи Rev Pharmacol Toxicol 40, 581-616.

van Duijn MM, van den Zee J, VanSteveninck J \& van den Broek PJA (1998) Ascorbate stimulates ferricyanide reduction in HL-60 cells through a mechanism distinct from the NADH-dependent plasma membrane reductase. J Biol Chem 273, 13415-13420.

van Dyke BR \& Saltman P (1996) Hemoglobin: a mechanism for the generation of hydroxyl radicals. Free Rad Biol Med 20, 985-989.

Williams RJ, Spencer JPE \& Rice Evans C (2004) Flavonoids: antioxidants or signaling molecules? Free Rad Biol Med 7, 838-849. 